\title{
A Co-evolutionary Multi-agent System for Multi-modal Function Optimization
}

\author{
Rafał Dreżewski \\ Department of Computer Science \\ AGH University of Science and Technology, Kraków, Poland \\ drezew@agh .edu.pl
}

\begin{abstract}
Niching methods for evolutionary algorithms are used in order to locate all desired peaks of multi-modal landscape. Co-evolutionary techniques are aimed at overcoming limited adaptive capacity of evolutionary algorithms resulting from the loss of useful diversity of population. In this paper the idea of niching co-evolutionary multi-agent system (NCoEMAS) is introduced. In such a system the niche formation phenomena occurs within one of the preexisting species as a result of coevolutionary interactions. Also, results from runs of NCoEMAS against Rastrigin function and the comparison to other niching techniques are presented.
\end{abstract}

\section{Introduction}

Terms Evolutionary Computation (EC) and Evolutionary Algorithms (EAs) cover a wide range of global search and optimization techniques based on analogies to natural evolution. Evolutionary algorithms (EAs) have demonstrated in practice efficiency and robustness as global optimization techniques. However, they often suffer from premature loss of population diversity what results in premature convergence and may lead to locating local optima instead of a global one. What is more, both the experiments and analysis show that for multi-modal problem landscapes a simple EA will inevitably locate a single solution [10]. If we are interested in finding multiple solutions of comparable fitness, some multimodal function optimization techniques should be used. Niching methods for EAs [10] are aimed at forming and stably maintaining niches (species) throughout the search process, thereby allowing to identify most of desired peaks of multi-modal landscape.

The loss of diversity also limits the adaptive capacities of EAs in dynamic environments. Co-evolutionary techniques are aimed at improving adaptive capacities and introducing open-ended evolution into EAs [11.

This paper introduces the idea of niching co-evolutionary multi-agent system (NCoEMAS), which opens new possibilities of modeling biological speciation mechanisms based on predator-prey and host-parasite co-evolution, sexual preferences, competition for limited resources, and geographical isolation. Also, results from runs of sample NCoEMAS against Rastrigin test function are presented and the comparison to other niching techniques is made. 


\section{Niching Techniques}

Various mechanisms have been proposed to form and stably maintain species throughout the search process. Most of these techniques allow niche formation through the implementation of crowding, fitness sharing or some modifications of these mechanisms.

In the crowding (CR) technique [5] each generation, a proportion of the population $G$ (generation gap) is selected for reproduction. For each offspring $C F$ (crowding factor) individuals are selected at random. The most similar individual, according to a similarity metric, is then replaced by the offspring.

Mahfoud developed niching mechanism called deterministic crowding (DC) 9]. In his technique children are directly compared to their parents. Parent is replaced only if the competing child has higher fitness.

Fitness sharing (FS) was first introduced by Holland and further developed by Goldberg and Richardson [7. In sharing technique each individual is considered to be the center of a niche with radius $\sigma_{s h}$. Fitness of each individual is reduced for every other individual, which lives in its niche, in a proportion to their similarity. The reduced fitness of an individual $i$ is given by $f_{i}^{\prime}=\frac{f_{i}}{m_{i}}$, where $f_{i}$ is its raw fitness and $m_{i}$ is the niche count. The niche count is given by $m_{i}=\sum_{j=1}^{n} \operatorname{sh}\left(d_{i j}\right)$, where $d_{i j}$ is the distance between individual $i$ and individual $j$, determined by a similarity metric. The sharing function is given by

$$
\operatorname{sh}(d)= \begin{cases}1-\left(\frac{d}{\sigma_{s h}}\right)^{\alpha} & \text { if } d<\sigma_{s h} \\ 0 & \text { otherwise }\end{cases}
$$

where $\alpha$ is a constant that regulates the shape of the sharing function (it is commonly set to 1 ).

Co-evolutionary shared niching (CSN) technique was developed by Goldberg and Wang [8]. Their technique was inspired by the economic model of monopolistic competition. The customer population is the usual population of candidate solutions. The businessman population evolve to obtain largest payoff (best cover the peaks in multi-modal domain). Customer $c$ is served by businessman $b$ if $b$ is the nearest businessman according to some similarity measure (Hamming distance of binary strings is used). The modified customer fitness is $f^{\prime}(c)=\left.\frac{f(c)}{m_{b, t}}\right|_{c \in C_{b, t}}$, where $C_{b, t}$ is the set of customers that are served by businessman $b$ at generation $t$, and $m_{b, t}=\left\|C_{b, t}\right\|$ is the number of customers that businessman $b$ serves at generation $t$. The modified businessman fitness is $\phi(b)=\sum_{c \in C_{b, t}} f(c)$.

\section{Previous Research in Co-evolutionary Algorithms}

In classical EAs each individual in the population is considered to be a potential solution of the problem being solved. The fitness of each individual depends only on how well it solves the problem. Selection pressure causes that better fit 
individuals have the greater chance to survive and/or reproduce and the less fit ones have the smaller chance.

In co-evolutionary systems the fitness of each individual depends not only on the quality of solution to the given problem but also on other individuals' fitness. As the result of ongoing research many co-evolutionary techniques have been proposed. Generally, each of these techniques belongs to one of two classes: "Competitive Fitness Functions" (CFF) or multi-population [11].

In $\mathrm{CFF}$ based systems two (or more) individuals compete in a game and their "Competitive Fitness Functions" are calculated based on their relative performance in that game 4. Each time step given individual competes with different opponents, so its fitness value varies. Because in such systems an individual's fitness depends on other individuals' fitness, they are co-evolutionary in nature.

The second group consists of systems that use multiple populations. In such systems a problem is decomposed into sub-problems and each of them is then solved by different EA 12 . Each individual is evaluated within a group of randomly chosen individuals coming from different sub-populations. Its fitness value depends on how well the group solved the problem and on how well the individual assisted in the solution.

Although co-evolutionary techniques are aimed at overcoming limited adaptive capacity of evolutionary algorithms resulting from the loss of useful diversity of population, they are not very often applied in the field of multi-modal optimization. In fact, to our best knowledge, only one niching technique based on co-evolution was developed (CSN).

In the following sections we will present the idea of co-evolution realized in multi-agent system, which allows us to define many co-evolutionary interactions that exist in nature.

\section{The Idea of Co-evolutionary Multi-agent Systems}

The main idea of evolutionary multi-agent system (EMAS) is the modeling of evolution process in multi-agent system (MAS) [3]. Co-evolutionary multiagent system (CoEMAS) allows co-evolution of several species of agents [6]. CoEMAS can be applied, for example, to multi-objective optimization and multi-modal function optimization (niching co-evolutionary multi-agent system - NCOEMAS).

In CoEMAS several (usually two) different species co-evolve. One of them represents solutions. The goal of the second species is to cooperate (or compete) with the first one in order to force the population of solutions to locate Pareto frontier or proportionally populate and stably maintain niches in multi-modal domain.

It seems that CoEMAS is especially suited for modeling different co-evolutionary interactions (resource competition, predator-prey and host-parasite co-evolution, sexual preferences, etc.) 


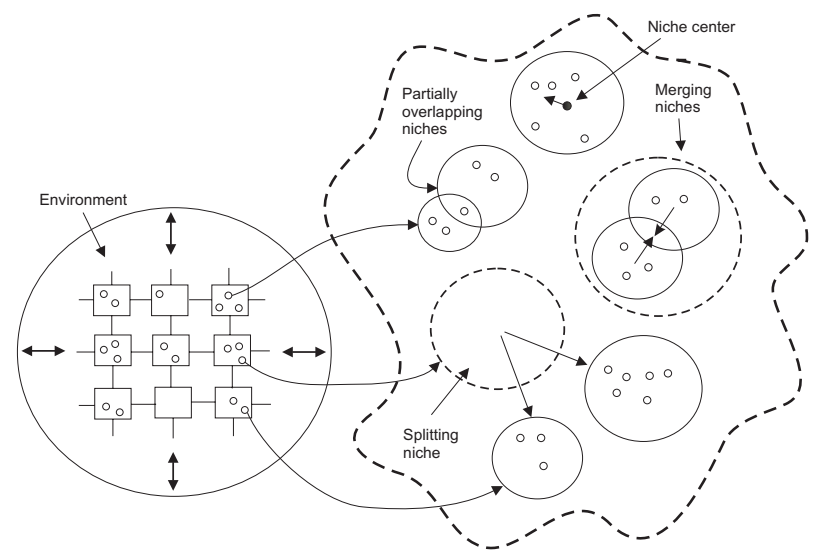

Fig. 1. Niching co-evolutionary multi-agent system used in experiments

\section{Sample Niching Co-evolutionary Multi-agent System}

In figure 1 sample co-evolutionary multi-agent system for multi-modal optimization is presented. The topography of environment, in which individuals live, is graph with every node (place) connected with its four neighbors. Within the environment two co-evolving species: niches and solutions live. There exist resource in the environment which is given to the niches and then distributed between solutions, that live within each niche, proportionally to their fitness. The more solutions live within the niche the more resource is given to it. Every action (such as migration or reproduction) of individual costs some resource.

Niches can migrate within the environment and all solutions live within niches. Each time step every solution searches for the niche that is located on the same peak. Modified version of hill-valley function [13] is used in order to check if two individuals are located on the same peak. If there are no niches located on the same peak, solution creates new niche, which genotype is the copy of its own genotype (niche is split into two niches). Then each solution searches its niche for the reproduction partner. Reproduction takes place only when individuals have enough amount of resource. The genotypes of all individuals are real-valued vectors. Intermediate recombination [2] and mutation with self-adaptation [1] are used for solutions and special mutation for niches. Each time step the niche's genotype is mutated in such a way, that the resulting genotype is the center of gravity of solutions that belong to the niche (fitness of each individual serves as a weight value). Niches can merge if they are at the same place and are located on the same peak in multi-modal domain.

The system was applied to multi-modal function optimization and then compared to other niching techniques. 


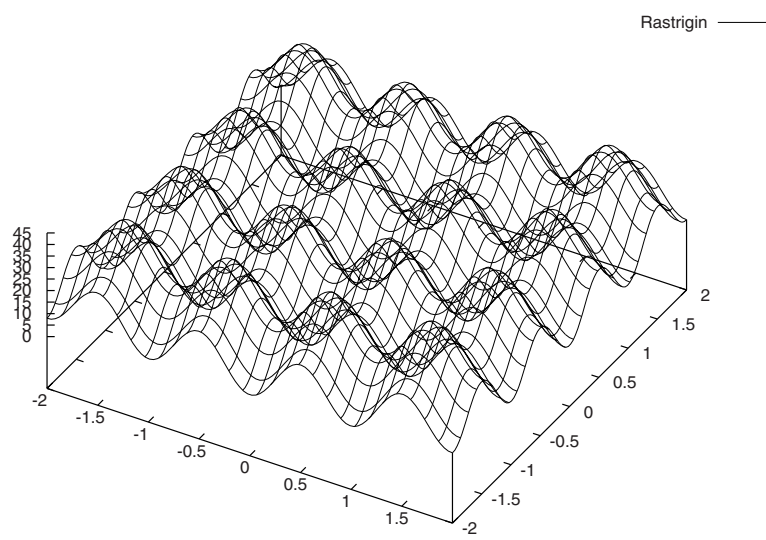

Fig. 2. Rastrigin function

\section{Simulation Experiments}

First simulation experiments were aimed at testing if NCoEMAS described in previous section is able to detect and stably maintain most of peaks in multimodal domain throughout the search process. Also, the comparison to other niching techniques such as DC and FS, and EMAS was made.

\subsection{Test Function}

In all experiments Rastrigin function was used as the test fitness landscape (see fig. 2). This is multi-modal function commonly used in studies of niching methods. Rastrigin function used in experiments is given by

$$
10 * n+\sum_{i=1}^{n}\left(x_{i}^{2}-10 * \cos \left(2 * \pi * x_{i}\right)\right) \quad x_{i} \in[-2.0,2.0] \text { for } i=1, \ldots, n
$$

where $n$ is the number of dimensions ( $n=2$ in all experiments). The function has 16 maxima for $x_{1}, x_{2} \in[-2.0,2.0]$.

\subsection{Results}

In this section the results from runs of NCoEMAS against Rastrigin function are presented. Also the comparison to other niching techniques is made.

Figures 3 and 4 show the location of NCoEMAS individuals in fitness landscape during the typical simulation. At the beginning there are only 5 niches (represented with black circles) and 5 solutions (represented with dots) with identical genotypes as niches. It can be seen that as the simulation goes on the 


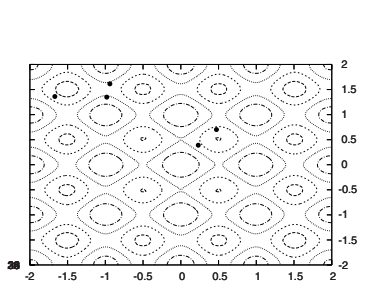

40
30
20
10

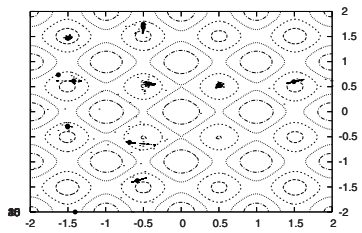

a)

b)

Fig. 3. The location of individuals in NCoEMAS during the 0th (a) and 100th (b) simulation step

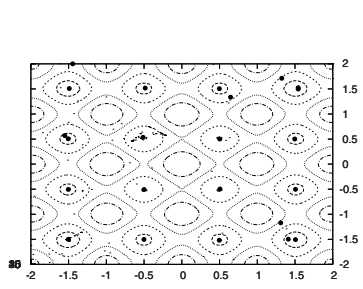

a)
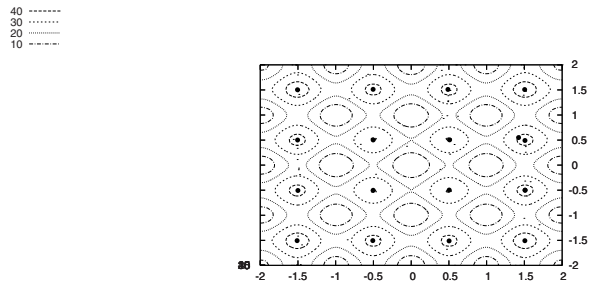

b)

Fig. 4. The location of individuals in NCoEMAS during the 1000th (a) and 5000th (b) simulation step

individuals reproduce and locate themselves near the centers of peaks in multimodal domain. What is more the subpopulations are stable, and do not disappear throughout the simulation. The population size self-adapts to the number of peaks in multi-modal domain.

Figure 5 shows the average number of located peaks from 10 simulations. The peak was classified as located when there was at least one individual closer than 0.05 to that peak. The experiments was made for four techniques: CoEMAS, EMAS, DC and FS. Each experiment was carried out with the different values of most important parameters (like initial population size, $\sigma_{s h}$ for FS, etc.)

CoEMAS stood relatively well when compared to other techniques. On the average, it stably maintained about 15 peaks starting from the smallest population of all techniques (between 5 and 10 individuals). DC started from 300-500 individuals, quickly located the same number of peaks but there was quite strong tendency to lose almost all peaks during the rest part of simulation. FS technique used almost the same initial population size as DC and detected and stably maintained about 8-9 peaks on the average. Simple EMAS was not able to stably populate more than one peak, although it started from much bigger population 


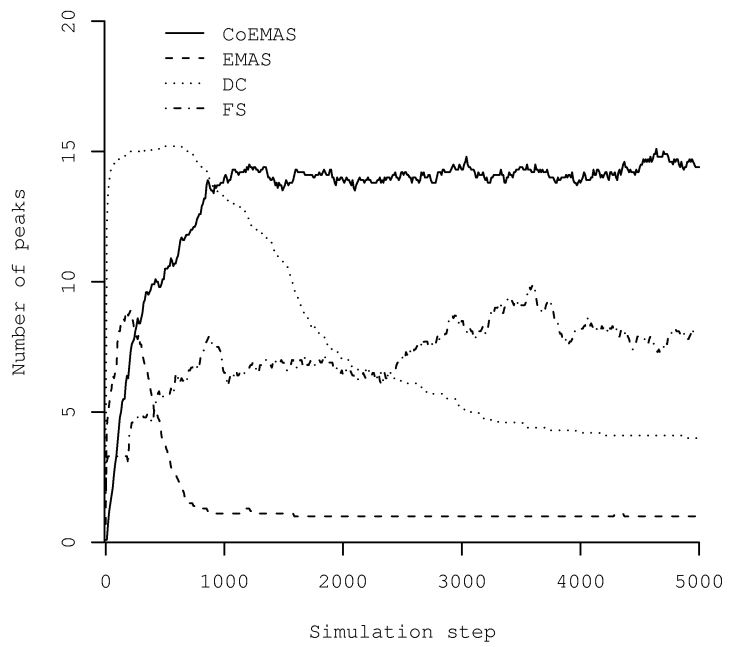

Fig. 5. The average number of detected peaks from 10 simulations

of about 150 individuals. It turned out that in case of multi-modal landscape it works just like simple EA.

To sum up, simple EMAS can not be applied to multi-modal function optimization without introducing special mechanisms such as co-evolution. FS and DC have some limitations as niching techniques, for example DC has the strong tendency to lose peaks during the simulation. The fact of relatively poor performance of DC was also observed in other works [14. CoEMAS is valid and promising niching technique but still more research is needed.

\section{Concluding Remarks}

The idea of co-evolutionary multi-agent system (CoEMAS) allows us to model many ecological co-evolutionary interactions between species such as resource competition, predator-prey and host-parasite co-evolution, sexual preferences, etc.

In this paper sample CoEMAS with two co-evolving species: niches and solutions was presented. This system was applied to multi-modal function optimization. It properly detected and maintained most of the peaks in multi-modal fitness landscape and, as presented preliminary results show, has proved to be the valid and promising niching technique. What is more, it turned out that presented system was able to detect and stably maintain more peaks of Rastrigin function than other classical niching techniques.

Future research will include more detailed comparison to other niching techniques, CoEMAS based on the mechanisms of predator-prey or host-parasite coevolution and sexual preferences. Also the parallel implementation of CoEMAS using MPI is included in future research plans. 


\section{References}

1. T. Bäck, D. Fogel, D. Whitley, and P. Angeline. Mutation. In T. Bäck, D. Fogel, and Z. Michalewicz, editors, Handbook of Evolutionary Computation. IOP Publishing and Oxford University Press, 1997.

2. L. Booker, D. Fogel, D. Whitley, and P. Angeline. Recombination. In T. Bäck, D. Fogel, and Z. Michalewicz, editors, Handbook of Evolutionary Computation. IOP Publishing and Oxford University Press, 1997.

3. K. Cetnarowicz, M. Kisiel-Dorohinicki, and E. Nawarecki. The application of evolution process in multi-agent world to the prediction system. In Proc. of the 2nd Int. Conf. on Multi-Agent Systems - ICMAS'96, Osaka, Japan, 1996. AAAI Press.

4. P. J. Darwen and X. Yao. On evolving robust strategies for iterated prisoner's dilemma. Lecture Notes in Computer Science, 956, 1995.

5. K. A. De Jong. An analysis of the behavior of a class of genetic adaptive systems. PhD thesis, University of Michigan, Ann Arbor, Michigan, USA, 1975.

6. R. Dreżewski. A model of co-evolution in multi-agent system. In V. Mař́k, J. Müller, and M. Pěchouček, editors, Multi-Agent Systems and Applications III, number 2691 in LNAI, pages 314-323, Berlin, Heidelberg, 2003. Springer-Verlag.

7. D. E. Goldberg and J. Richardson. Genetic algorithms with sharing for multimodal function optimization. In J. J. Grefenstette, editor, Proc. of the 2nd Int. Conf. on Genetic Algorithms, Hillsdale, NJ, 1987. Lawrence Erlbaum Associates.

8. D. E. Goldberg and L. Wang. Adaptive niching via coevolutionary sharing. Technical Report IlliGAL 97007, Illinois Genetic Algorithms Laboratory, University of Illinois at Urbana-Champaign, Urbana, IL, USA, 1997.

9. S. W. Mahfoud. Crowding and preselection revisited. In R. Manner and B. Manderick, editors, Parallel Problem Solving From Nature, 2, Amsterdam, 1992. Elsevier Science Publishers (North Holland).

10. S. W. Mahfoud. Niching methods for genetic algorithms. PhD thesis, University of Illinois at Urbana-Champaign, Urbana, IL, USA, 1995.

11. J. Morrison and F. Oppacher. A general model of co-evolution for genetic algorithms. In Int. Conf. on Artificial Neural Networks and Genetic Algorithms ICANNGA 99, 1999.

12. M. A. Potter and K. A. De Jong. Cooperative coevolution: An architecture for evolving coadapted subcomponents. Evolutionary Computation, 8(1), 2000.

13. R. K. Ursem. Multinational evolutionary algorithms. In P. J. Angeline, Z. Michalewicz, M. Schoenauer, X. Yao, and A. Zalzala, editors, Proceedings of the Congress on Evolutionary Computation, volume 3, pages 1633-1640, Mayflower Hotel, Washington D.C., USA, 6-9 1999. IEEE Press.

14. J.-P. Watson. A performance assessment of modern niching methods for parameter optimization problems. In W. Banzhaf, J. Daida, A. E. Eiben, M. H. Garzon, V. Honavar, M. Jakiela, and R. E. Smith, editors, Proceedings of the Genetic and Evolutionary Computation Conference, volume 1, pages 702-709, Orlando, Florida, USA, 13-17 1999. Morgan Kaufmann. 